\title{
Compostos bioativos e características físico-químicas de polpa de araticum in natura e pasteurizada
}

\author{
Bioactive compounds and physicochemical characteristics of in natura and \\ pasteurized araticum pulp
}

\author{
Elaine Carvalho de Morais ${ }^{1 *}$, Samira Gabrielle de Oliveira Patias ${ }^{1}$, Nayara Suzana da Silva Ferreira ${ }^{2}$, \\ Nágela Farias M. Picanço', Erika Cristina Rodrigues', Edgar Nascimento', \\ Rozilaine Aparecida Pelegrine Gomes de Faria ${ }^{1}$
}

${ }^{1}$ Instituto Federal de Educação Ciência e Tecnologia de Mato Grosso, Departamento de Pós-graduação em Ciência e Tecnologia de Alimentos, Cuiabá/MT - Brasil 2Instituto Federal de Educação Ciência e Tecnologia de Mato Grosso, Departamento de Engenharia de Alimentos, Cuiabá/MT - Brasil

\section{*Corresponding Author}

Elaine Carvalho de Morais, Instituto Federal de Educação Ciência e Tecnologia de Mato Grosso, Departamento de Pós-graduação em Ciência e Tecnologia de Alimentos, Campus Cuiabá-Bela Vista, Rua Juliano Costa Marques, s/n, Bairro Bela Vista, CEP: 78050-560, Cuiabá/MT - Brasil, e-mail: elainemorais.morais@gmail.com

Cite as: Bioactive compounds and physicochemical characteristics of in natura and pasteurized araticum pulp. Braz. J. Food Technol., v. 20, e2016142, 2017.

\section{Resumo}

O objetivo deste trabalho foi avaliar o potencial antioxidante, o teor de compostos fenólicos e de carotenoides de polpa de araticum in natura e pasteurizada, e avaliar o efeito da pasteurização nas suas características físico-químicas. A polpa de araticum in natura foi adquirida em Aragarças/GO e dividida em dois lotes: polpa in natura e polpa pasteurizada. As polpas foram analisadas em triplicata, em relação às variáveis: pH, acidez titulável (AT), vitamina C, sólidos solúveis (SS), relação entre SS/AT, atividade de água, cor, atividade antioxidante radicalar, compostos fenólicos e carotenoides, além da composição centesimal da polpa pasteurizada. A polpa in natura apresentou 0,98 de atividade de água e 9,57 ${ }^{\circ} \mathrm{Brix}$ de sólidos solúveis, e a polpa pasteurizada apresentou valores de 0,99 e 9,23 Brix para atividade de água e sólidos solúveis, respectivamente. Os resultados mostraram que as polpas apresentaram relevantes propriedades antioxidantes, expressas na capacidade de reduzir em $50 \%$ o radical DPPH ( $\left(\mathrm{C}_{50} \mathrm{mg} / \mathrm{mL}\right.$ ). Com relação aos compostos fenólicos, ambas apresentaram valores próximos de 227,13 e 207,18 mg GAE/100 g - para polpa in natura e pasteurizada, respectivamente. A polpa pasteurizada apresentou $8,21 \mu \mathrm{g} / \mathrm{g}$ de $\beta$ caroteno e 4,97 $\mathrm{\mu g} / \mathrm{g}$ de $\alpha$ caroteno. Conclui-se que as polpas analisadas apresentam um elevado conteúdo de compostos bioativos e que o processo de pasteurização não interferiu nas características físico-químicas nem nos compostos bioativos analisados da polpa in natura.

Palavras-chave: Marolo; Tratamento térmico; Fruto do Cerrado; Annona crassiflora.

\section{Abstract}

The objective of this study was to evaluate the antioxidant potential and the phenolic compound and carotenoid contents of in natura and pasteurized araticum pulp, and evaluate the effect of pasteurization on the physicochemical characteristics. The in natura araticum pulp was acquired in Aragarças/GO, Brazil and divided into two portions: in natura and pasteurized pulp. The pulps were analysed in triplicate in relation to the variables: $\mathrm{pH}$, titratable acidity, vitamin $\mathrm{C}$, soluble solids, water activity, colour, radical antioxidant activity, phenolic and carotenoid compounds, as well as the proximate composition of the pasteurized pulp. The in natura pulp presented 0.98 of water activity and $9.57^{\circ} \mathrm{Brix}$ of soluble solids, however the pasteurized pulp showed values of 0.99 and $9.23^{\circ}$ Brix for water activity and soluble solids, respectively. The results showed that the pulp had significant antioxidant properties, expressed as the ability to reduce the DPPH value by $50 \%\left(\mathrm{IC} \mathrm{C}_{50} \mathrm{mg} / \mathrm{ml}\right)$. With respect to the phenolic compounds, both presented similar values of 227.13 and $207.18 \mathrm{mg} \mathrm{GAE} / 100 \mathrm{~g}$ of in natura and pasteurized pulps, respectively. The pasteurized pulp presented $8.21 \mu \mathrm{g} / \mathrm{g}$ of $\beta$-carotene and $4.97 \mu \mathrm{g} / \mathrm{g}$ of $\alpha$-carotene. It was concluded that the pulps analysed presented high contents of bioactive compounds and that pasteurization did not affect the physicochemical characteristics or bioactive compounds of the in natura pulp.

Keywords: Marolo; Heat treatment; Fruit of the Cerrado; Annona crassiflora. 


\section{Introdução}

O araticum (Annona crassiflora), também conhecido como marolo ou pinha-do-cerrado, é uma fruta típica do Cerrado brasileiro, pertencente à família Annonaceae, sendo bastante apreciado pela população local. Sua polpa doce de sabor característico e aroma forte pode ser consumida in natura ou na forma de doces, geleias, sucos, iogurte ou sorvete (ALMEIDA et al., 2008).

O consumo regular de frutas tornou-se um forte aliado da busca por uma alimentação saudável, devido ao valor nutritivo e por ser fonte de fibras, vitaminas, minerais e antioxidantes. Estudos mostram que populações que têm como hábito o consumo regular de frutos e/ou outros alimentos ricos em compostos antioxidantes apresentam baixa incidência de doenças degenerativas, provocadas por compostos provenientes do estresse oxidativo (ROESLER et al., 2007).

A oferta de frutas no período de entressafra é uma necessidade da demanda do consumidor e diversos métodos podem ser utilizados para conservação de polpa de frutas, dentre os quais a pasteurização. A pasteurização é um dos métodos mais conhecidos e eficientes, pois visa à inativação das enzimas e à destruição dos microrganismos, preservando as características físicas, químicas e nutricionais da fruta (ELEZ-MARTÍNEZ; MARTÍN-BELLOSO, 2007), e possibilita oferecer a polpa do fruto no período de entressafra.

As frutas, além de estarem associadas a uma dieta saudável, fornecem componentes importantes, que atuam nas funções básicas do nosso organismo, como, por exemplo, o ácido ascórbico, o betacaroteno e a vitamina $E$, fontes de compostos bioativos ligados à prevenção de doenças. Alguns frutos do Cerrado vêm sendo estudados na busca de encontrar aquele que possua, na sua composição, substância com atividade antioxidante, como carotenoides, vitamina $\mathrm{C}$ e flavonoides (CARDOSO et al., 2013; ROCHA et al., 2013).

Os antioxidantes são compostos que agem impedindo e/ou diminuindo a formação de radicais livres ou espécies reativas não radicalares, combatendo o processo oxidativo. Tais radicais ou espécies reativas são compostos produzidos pelo metabolismo normal do corpo e que, em grande quantidade, podem provocar danos extensivos (SANTOS et al., 2008).

A procura por antioxidantes naturais tem aumentado bastante nos últimos anos e cada vez mais pesquisadores estão à procura de frutas nativas que apresentem essa propriedade. Essas frutas podem conter compostos em quantidade mínima suficiente para proteger as células contra processos degenerativos (NEVES, 2012; HINNEBURG et al., 2006; JAYAPRAKASHA; RAO, 2000).

A incorporação na dieta alimentar de frutas regionais estimula a oferta de novos produtos, buscando melhorar a qualidade nutricional da alimentação dessas comunidades locais, pois estas conhecem a fruta consumida na região. No entanto, a temperatura da pasteurização pode comprometer a atividade dos compostos encontrados nessas frutas, bem como alterar as características físico-químicas da polpa. Relatos na literatura sobre a influência da temperatura da pasteurização sobre teor de carotenoides e vitamina C são contraditórios (PLAZA et al., 2006; LEE; COATES, 2003), pois tal influência depende do tipo de matriz alimentar. Note-se que a temperatura da pasteurização pode influenciar a cor, bem como o sabor do produto final.

É possível que o processo de pasteurização possa interferir nas propriedades físico-químicas da polpa. Diante do exposto, este trabalho teve por objetivo avaliar o potencial antioxidante, o teor de compostos fenólicos e de carotenoides de polpa de araticum in natura e pasteurizada, e avaliar o efeito da pasteurização nas suas características físico-químicas.

\section{Material e métodos}

\subsection{Matéria-prima}

A polpa de araticum in natura foi obtida no comércio local de Aragarças/GO, em janeiro de 2014, tendo sido embalada a vácuo em sacos plástico de polietileno, transportada em caixa térmica para o Instituto Federal de Educação, Ciência e Tecnologia de Mato Grosso - Campus Cuiabá - Bela Vista, onde ficaram armazenadas a $-18^{\circ} \mathrm{C}$, até o momento da realização das análises. As polpas foram divididas em dois lotes e denominadas polpa in natura e polpa pasteurizada.

Para a obtenção da polpa pasteurizada, a amostra foi submetida a tratamento térmico de $85^{\circ} \mathrm{C}$ por três minutos, em banho-maria, e resfriada em banho de gelo, sendo posteriormente armazenada em recipientes fechados de polietileno a $-18^{\circ} \mathrm{C}$, até o momento das análises.

\subsection{Análise físico-química das polpas}

Amostras da polpa in natura e pasteurizada foram descongeladas em refrigeração a $4{ }^{\circ} \mathrm{C}$ e submetidas às análises de: $\mathrm{pH}$, por potenciometria direta, utilizando-se um pHmetro digital de bancada, marca HANNA INSTRUMENTS ${ }^{\circledR}$, modelo HI 2221, previamente calibrado com soluções tampão 4 e 7, de acordo com o método n. 981.12 da Association of Official Analytical Chemists (LATIMER JUNIOR, 2012); acidez titulável (AT), determinada por titulação de neutralização com $\mathrm{NaOH} \mathrm{0,1} \mathrm{mol/L,} \mathrm{com} \mathrm{os}$ resultados expressos em grama de ácido málico por $100 \mathrm{~g}$ de polpa; sólidos solúveis (SS), teor medido diretamente em refratômetro digital de bancada (INSTRUTHERM, modelo RTD - 95), sendo os resultados expressos em 'Brix, segundo método n. ${ }^{\circ} 932.12$ da AOAC (LATIMER JUNIOR, 2012); relação matemática entre o teor de sólidos 
solúveis e a acidez titulável (SS/AT); atividade de água (Aw), medida em aparelho digital da marca DECAGON ${ }^{\circledR}$, modelo Aqualab 4TE, segundo método n. ${ }^{\circ} 978.18$ da AOAC (LATIMER JUNIOR, 2012); o teor de vitamina C (ácido ascórbico) foi determinado pelo método de Tillmans, sendo os resultados expressos em miligrama de ácido ascórbico por $100 \mathrm{~g}$ de polpa (IAL, 2008). Todas as análises foram realizadas em triplicata.

A determinação de cor nas amostras foi realizada utilizando-se um espectrofotômetro da marca KONICA MINOLTA ${ }^{\circledR}$, modelo CM-700D (Minolta Co., Ltd., Tóquio, Japão), na escala $L^{*}, a^{*}$ e b* do sistema CIELab, calibrado com um padrão branco (placa de calibração), seguindo as instruções do fabricante, sendo as medidas realizadas em três pontos distintos da amostra, com três medições cada. O valor de $L^{*}$ determina a posição do ponto sobre o eixo vertical de claridade; a coordenada $a^{*}$ refere-se ao ponto sobre o eixo $a^{*}(-)$ verde (+) vermelho, e o valor de $b^{*}$ correspondente ao ponto sobre o eixo (-) azul (+) amarelo. Os índices de saturação $C^{*}$, tonalidade $h^{*} e$ diferença da cor $\Delta \mathrm{E}^{*}$ foram calculados a partir dos índices de cromaticidade $\left(L^{*}, a^{*}\right.$ e $\left.b^{*}\right)$, conforme as Equações 1, 2 e 3 (RAMOS; GOMIDE, 2007; LEE; COATES, 2003).

$$
C^{*}=(a * 2+b * 2) 1 / 2
$$

$\mathrm{h}^{*}=\tan ^{-1}\left(\mathrm{~b}^{*} / \mathrm{a}^{*}\right)$

$$
\Delta \mathrm{E}^{*}=\left(\Delta \mathrm{L}^{*} 2+\Delta \mathrm{a} * 2+\Delta \mathrm{b}^{*} 2\right)^{1 / 2}
$$

\subsection{Obtenção dos extratos para determinação da atividade antioxidante radicalar e de compostos fenólicos totais}

Para análise dos compostos fenólicos totais e da atividade antioxidante radicalar, obteve-se extrato conforme metodologia proposta por Rufino et al. (2010). A extração foi realizada utilizando-se $1 \mathrm{~g}$ (para análise de fenólicos totais) e $5 \mathrm{~g}$ (para análise da atividade antioxidante radicalar) de cada amostra. À amostra foram acrescentados $40 \mathrm{~mL}$ de metanol: água (50:50, v:v), sendo esse preparo deixado em repouso por $60 \mathrm{~min}$; em seguida, a amostra foi centrifugada (centrífuga modelo UNIVERSAL 320R) a 6088 força g ou RCF - raio do rotor $151 \mathrm{~mm}$, por $40 \mathrm{~min}$, sendo o sobrenadante reservado em balão volumétrico fechado e protegido da luz, e o resíduo restante submetido à nova extração com solução aquosa de acetona 70\% (v:v). O sobrenadante foi acrescentado a um balão volumétrico de $100 \mathrm{~mL}$ e o volume completado até a marca de aferição com água destilada.

\subsection{Atividade antioxidante radicalar}

A atividade antioxidante radicalar foi realizada pelo método de DPPH, baseado na captura do radical DPPH (2,2-difenil-1-picril-hidrazila) por compostos antioxidantes.
A partir dos extratos aceto-hidro-alcoólico obtidos, foram realizadas cinco diluições diferentes em metanol (P.A), com concentrações de 10, 20, 30, 35 e $45 \mathrm{mg} / \mathrm{mL}$, sendo então transferidas alíquotas de $0,1 \mathrm{~mL}$ de cada diluição dos extratos para tubos de ensaio contendo 3,9 $\mathrm{mL}$ da solução de DPPH 0,06 mM. A leitura da absorbância foi realizada em espectrofotômetro de marca SHIMADZU ${ }^{\circledR}$, modelo UV - 1800, em comprimento de onda de $517 \mathrm{~nm}$, ao abrigo da luz, após 30 min do início da reação.

A capacidade de descoloração do radical DPPH foi comparada com o controle $(3,9 \mathrm{~mL}$ de DPPH e 0,1 mL de metanol), estabelecendo a porcentagem de inibição do radical conforme a Equação 4:

$\%$ de inibição $=\left[\left(\begin{array}{l}\text { AbsCONTROLE }- \\ \text { AbsAMOSTRA }\end{array}\right) /\right.$ AbsCONTROLE $] \times 100$

em que: AbsCONTROLE: absorbância da solução controle (metanol P.A); AbsAMOSTRA: absorbância da amostra.

$\mathrm{O}$ valor do $\mathrm{IC}_{50}$ (quantidade de amostra necessária para reduzir em 50\% a concentração inicial do radical DPPH) foi obtido através dos resultados de porcentagem de inibição em diferentes concentrações (10, 20, 30, 35 e 45 mg/mL), de forma a traçar uma curva linear e obter a equação da reta para o cálculo do $\mathrm{IC}_{50}$.

\subsection{Compostos fenólicos totais}

A determinação de compostos fenólicos totais foi realizada através do método espectrofotométrico, com o reagente Folin-Ciocalteu, conforme descrito por Obanda et al. (1997). Foram utilizadas alíquotas de $1 \mathrm{~mL}$ do extrato aceto-hidro-alcoólico, $2 \mathrm{~mL}$ de água destilada, 2 mL da solução aquosa de carbonato de sódio 20\% (m:v) e $1 \mathrm{~mL}$ do reagente Folin-Ciocalteu (1:3, v:v). Após 30 min, a absorbância foi lida em espectrofotômetro SHIMADZU modelo UV - 1800, em comprimento de onda de $700 \mathrm{~nm}$. Os resultados foram expressos em miligrama de equivalente de ácido gálico (GAE) por $100 \mathrm{~g}$ do extrato da polpa, obtidos por meio da equação da reta ( $y=0,0169 x-0,0029$; $\left.\mathrm{R}^{2}=0,9983\right)$ da curva analítica construída com ácido gálico nas concentrações de 0, 10, 20, 30, 40 e 50 mg/mL.

\subsection{Carotenoides}

Para análise de carotenoides ( $\alpha$-caroteno e $\beta$-caroteno), foi adicionado $1,5 \mathrm{~g}$ de celite (P.A) e macerado com aproximadamente $1 \mathrm{~g}$ de polpa de cada lote; em seguida, foram homogeneizados com $25 \mathrm{~mL}$ de acetona (P.A) e filtrado a vácuo em funil de Büchner. O procedimento de extração foi repetido por mais duas vezes no resíduo, até a descoloração completa da polpa, indicando completa extração. Logo a seguir, o extrato foi transferido para um funil de separação contendo éter de petróleo (P.A); homogeneizou-se e adicionaram-se $150 \mathrm{~mL}$ de água destilada para separação das fases (superior: carotenoides 
em éter de petróleo; inferior: acetona-água), sendo a fase inferior descartada. A parte superior foi coletada e teve completado o volume para $50 \mathrm{~mL}$ com éter de petróleo (P.A). Posteriormente, foi feita a leitura da absorbância em espectrofotômetro de marca SHIMADZU modelo UV - 1800 em dois comprimentos de onda (453 e 444 nm) (IAL, 2008; PACHECO et al., 2011).

Para o cálculo do $\beta$-caroteno $(\mu \mathrm{g} / \mathrm{g})$, foi utilizada a Equação 5:

$\beta$ carotenos $(\mu \mathrm{g} / \mathrm{g})=\left(\begin{array}{l}\text { Absorbância do } \\ \text { extrato } \times 50 \times 104\end{array}\right) /\left(\begin{array}{c}2592 \times \text { massa } \\ \text { da amostra }\end{array}\right)$

E para o cálculo do $\alpha$-caroteno $(\mu \mathrm{g} / \mathrm{g})$, foi utilizada a Equação 6:

$\alpha$ carotenos $(\mu \mathrm{g} / \mathrm{g})=\left(\begin{array}{l}\text { Absorbância do } \\ \text { extrato } \times 50 \times 104\end{array}\right) /\left(\begin{array}{c}2400 \times \text { massa } \\ \text { da amostra }\end{array}\right)$

\subsection{Composição centesimal}

A polpa pasteurizada foi submetida à análise de composição centesimal, de acordo com as normas analíticas da AOAC (LATIMER JUNIOR, 2012). O teor de umidade foi quantificado pelo método gravimétrico n. ${ }^{\circ}$ 920.151, em estufa (FANEM ${ }^{\circledR}$ 520, Modelo A-HT); cinzas, por calcinação em mufla (FORNITEC ${ }^{\circledR}$, modelo MDS) a $550{ }^{\circ} \mathrm{C}$ (método 920.153); proteína, pelo método de Kjeldahl - TECNAL ${ }^{\circledR}$, modelo TE-0363 (método n. ${ }^{\circ}$ 928.08); lipídeos, por extração em aparelho Soxhlet TECNAL ${ }^{\circledR}$, modelo TE-044-5 (método n. ${ }^{\circ}$ 991.36); para glicídios redutores em glicose, foi utilizado o método que se baseia na redução de um volume conhecido do reagente cobre alcalino (Fehling) a óxido cuproso, e os glicídios não redutores em sacarose foram determinados após hidrolise ácida (método n. ${ }^{\circ} 31.034-6$ ).

O valor energético total da polpa pasteurizada foi calculado segundo os valores de conversão Atwater, utilizando-se $4 \mathrm{kcal} / \mathrm{g}$ para proteínas e carboidratos, e $9 \mathrm{kcal} / \mathrm{g}$ para lipídeos (MERRIL; WATT, 1973).

Todos os dados obtidos foram submetidos à análise de estatística descritiva e os resultados foram expressos em valores médios \pm desvio padrão, para cada variável.

\section{Resultados e discussão}

Os valores de $\mathrm{pH}$ e da acidez encontrados para as duas polpas foram similares (Tabela 1), não apresentando alteração com o tratamento térmico. Pimenta et al. (2014) e Cardoso et al. (2013) encontraram resultados semelhantes para polpas não tratadas termicamente, apresentando valores médios de $\mathrm{pH}$ de 4,45 e 4,71, respectivamente.

Em relação à acidez, Damiani et al. (2011) analisaram a porcentagem de acidez do araticum em diferentes ácidos orgânicos e constataram que o que mais se sobressaiu foi o ácido málico, apresentando teor de 958,5 $\mu \mathrm{g} / \mathrm{g}$. Essas diferenças encontradas podem estar relacionadas ao estádio de maturação dos frutos (Mosca et al., 2006), sugerindo que a temperatura ou o tempo da pasteurização não exerceu influência sobre a acidez da amostra.

A polpa de araticum in natura apresentou valor de sólidos solúveis (SS) superior ao apresentado pela pasteurizada; tal fato pode ser devido ao processo de pasteurização, que leva à perda de água, e a outros eventos que ocorrem durante a pasteurização. Apesar de a polpa in natura apresentar um valor elevado em comparação com a polpa pasteurizada, as mesmas possuem valores abaixo dos encontrados na literatura.

Braga Filho et al. (2014) analisaram os frutos do araticunzeiro provenientes de várias localidades e observaram valores médios de sólidos solúveis de 18,91 Bix. Porém, é importante salientar que o teor de SS depende do grau de maturação e é variável entre regiões, entre os cultivares e até mesmo entre as porções do mesmo fruto (CHITARRA; CHITARRA, 2005; PIMENTA et al., 2014).

Não há Padrão de Identidade e Qualidade (PIQ) para polpa de frutos de araticum. No entanto, para a polpa da graviola (Annona muricata L.), que pertence à mesma família do araticum, há PIQ estabelecido pela legislação, o qual determina o valor mínimo de 9 Brix (BRASIL, 2000). Por serem frutos que apresentam a mesma classificação botânica para gênero da espécie, pode-se afirmar que ambas as polpas apresentam teores de sólidos solúveis muito próximos, possibilitando interesse da indústria de alimentos em explorar o fruto do araticunzeiro típico do Cerrado de Mato Grosso, colaborando, assim, para os programas de preservação da espécie.

Tabela 1. Características físico-químicas da polpa de araticum (Annona crassiflora) in natura e pasteurizada, com suas respectivas médias \pm desvio padrão.

\begin{tabular}{lcc}
\multicolumn{1}{c}{ Características físico-químicas } & Polpa in natura & Polpa pasteurizada \\
pH & $4,45 \pm 0,01$ & $4,45 \pm 0,01$ \\
Sólidos Solúveis - SS ( ${ }^{\circ}$ Brix) & $9,57 \pm 0,26$ & $9,23 \pm 0,19$ \\
AT (g de ácido málico/100 g) & $0,30 \pm 0,001$ & $0,30 \pm 0,002$ \\
SS/AT & $32,26 \pm 1,56$ & $31,49 \pm 1,22$ \\
Vitamina C (mg/100 g) & $5,27 \pm 1,01$ & $5,75 \pm 0,04$ \\
Atividade de água & $0,98 \pm 0,001$ & $0,99 \pm 0,001$ \\
\hline
\end{tabular}


A relação SS/AT apresentou valor elevado para polpa in natura e, quanto maior essa relação, maior a estabilidade entre os açúcares e os ácidos orgânicos, tornando essa relação de grande importância para avaliação do sabor e deixando o fruto mais atrativo (CHITARRA; CHITARRA, 2005). Apesar de os valores médios da relação SS/AT se apresentarem mais baixos, em comparação com os obtidos por Pimenta et al. (2014), é possível que a polpa adquirida estivesse aquém do estádio ideal de maturação, uma vez que se trata de uma fruta silvestre e não domesticada, com efeitos de maturação em épocas diferentes. Dessa forma, para que o produtor possa obter ganho econômico, é preciso que ele atente para o fato de que, muitas vezes, a colheita dos frutos pode envolver frutos em estádios de maturação diferente, impactando na relação SS/AT final da polpa. É importante ressaltar que, conforme observado por Bianco e Pitelli (1986), a frutificação do araticum pode ocorrer entre setembro e janeiro, sem especificar o período de intensidade do fenômeno.

As polpas apresentaram valores de atividade de água de 0,98 e 0,99 para polpa in natura e pasteurizada, respectivamente.

Em geral, as polpas de frutas apresentam elevada atividade de água ( $>0,95)$, potencial de oxirredução elevado e pH baixo. Entre esses fatores, a acidez desempenha um fator na inibição da microbiota deteriorante, sendo os bolores e fungos filamentosos os mais resistentes a condições adversas com baixa atividade de água, o que pode levar à deterioração, principalmente em frutas frescas (FRANCO; LANDGRAF, 2008).

$O$ teor de vitamina $C$ foi superior para a polpa in natura; provavelmente, a pasteurização pode ter influenciado no teor dessa vitamina devido ao aquecimento. Como não há PIQ para a polpa de araticum, observa-se que o PIQ para polpa de fruta de graviola (Annona muricata L.), a qual pertence à mesma família, estabelece valor mínimo de $10 \mathrm{mg} / 100 \mathrm{mg}$ para vitamina $\mathrm{C}$. Em relação aos parâmetros de cor, a polpa pasteurizada apresentou os menores índices de $L^{*}(50,62 \pm 1,58), a^{*}(10,86 \pm 0,33)$ e $b^{*}(24,35 \pm 1,22)$ em relação à polpa in natura, mostrando que houve diminuição da concentração dos índices $\mathrm{a}^{*} \mathrm{e} \mathrm{b}^{*}$, respectivamente (Figura 1 ).

O processamento térmico, as reações térmicas sensíveis ao calor e a degradação de pigmentos podem favorecer a formação de compostos escuros, os quais podem estar associados à diminuição da luminosidade (ARÉVALO-PINEDO et al., 2013). Damiani et al. (2011) analisaram o parâmetro cor de polpas in natura do araticum e encontraram valores de $L^{*}(70,92 \pm 0,7), a^{*}(2,17 \pm 0,69)$ e b* $(33,90 \pm 0,71)$.

Os valores observados para $C^{*}$ foram de 65,12 para polpa in natura e 26,66 para pasteurizada, indicando que a polpa in natura apresentou coloração mais intensa, pois esse índice está relacionado com a saturação da amostra. $\mathrm{O}$ ângulo de tonalidade $\mathrm{h}^{*}$ é bastante utilizado por ser um atributo em que se classifica a cor em vários quadrantes, que vai variar de acordo com o ângulo que se encontra, podendo, dessa forma, a cor ser percebida como: vermelho, laranja, amarelo, verde, azul ou violeta. Assim, as polpas de araticum apresentaram valores de 65 para esse parâmetro e estão classificadas no primeiro quadrante da cor, no qual predominam as cores vermelha, amarela e laranja (RAMOS; GOMIDE, 2007). O resultado do $\Delta \mathrm{E}$ comparativo entre a polpa in natura e pasteurizada apresentou o valor de 9,4, indicando que a diferença da cor das amostras é facilmente detectável, mostrando uma percepção bastante clara de alteração na cor da polpa pasteurizada quando comparada com a polpa in natura. Amostras com diferenças de $\Delta \mathrm{E}^{*}$ acima de 2 faz com que se torne altamente perceptível a diferença na cor entre as duas amostras analisadas (LEE; COATES, 2003). Apesar de ter sido verificada diminuição na magnitude dos parâmetros $a^{*} e b^{*}$ entre as amostras in natura $\left(a^{*} 14,55\right.$ e $\left.b^{\star} 31,36\right)$ e pasteurizada ( $a^{*} 10,86$ e $\left.b^{*} 24,35\right)$, o parâmetro $b^{*}$ demonstrou valores positivos superiores em comparação com valor de $a^{*}$, fazendo a percepção tender para o amarelo, tanto na polpa in natura quanto na polpa pasteurizada. Essa tendência na mudança da coloração de vermelho para amarelo, a partir dos parâmetros $a^{*} e$ $b^{*}$ analisados, demonstra a necessidade de análise do produto final, buscando a melhor aceitabilidade pelo mercado consumidor.

As polpas apresentaram elevado poder antioxidante devido ao grau de descoloramento da solução de DPPH, que representa um alto poder antioxidante, possuindo baixos valores de $\mathrm{IC}_{50}$ (Tabela 2). Conforme classificação de Gregoris et al. (2013), as frutas podem apresentar três categorias relacionadas ao $\mathrm{IC}_{50}$ : boa, média e fraca, de acordo com os valores de $I C_{50}\left(I C_{50} \leq 100 \mathrm{ppm}\right)$; $\left(100 \mathrm{ppm}<\mathrm{IC}_{50} \leq 316 \mathrm{ppm}\right)$, e $\left(\mathrm{IC}_{50}>316 \mathrm{ppm}\right)$, respectivamente.

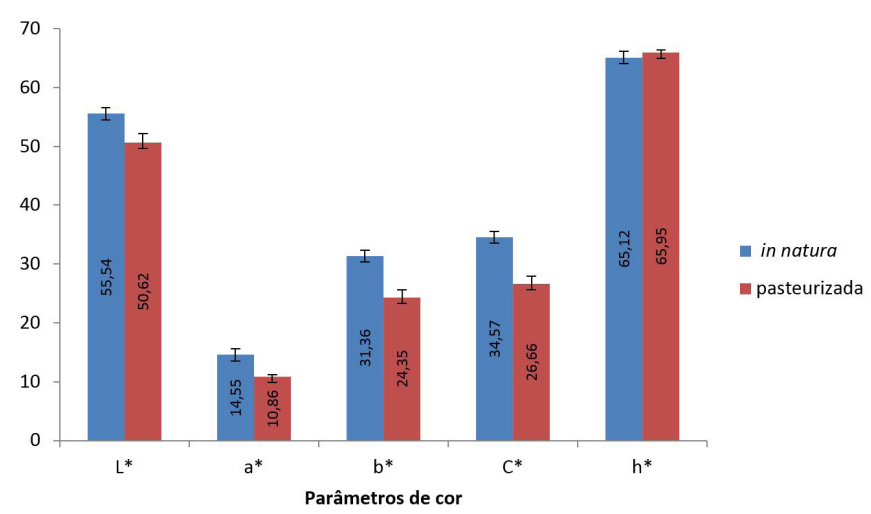

Figura 1. Média e desvio padrão para os parâmetros $L^{*}, a^{*}$, $\mathrm{b}^{\star}, \mathrm{C}^{\star} \mathrm{e} \mathrm{h}^{\star}$, referentes à análise de cor da polpa de araticum (Annona crassiflora) pasteurizada e in natura. 
O extrato da polpa que sofreu tratamento térmico apresentou valor de $\mathrm{IC}_{50}$ abaixo do apresentado pelo extrato da polpa in natura (Figura 2). Essa atividade antioxidante pode estar relacionada com o conteúdo de compostos fenólicos, carotenoides e ainda outros compostos bioativos, como taninos, flavonoides e ácidos carboxílicos, que podem estar presentes na polpa pasteurizada, pois o tratamento térmico influencia na decomposição desses compostos (SALES; WAUGHON, 2013; SOUZA-SARTORI et al., 2013).

Os valores encontrados neste estudo diferiram dos obtidos por Roesler et al. (2007), que encontraram $148,82 \mu \mathrm{g} / \mathrm{mL}$ para o extrato etanólico e 1.321,93 $\mathrm{\mu g} / \mathrm{mL}$ para o extrato aquoso em polpa de araticum em massa seca. Essas diferenças encontradas podem estar relacionadas com o tipo de extração, o tipo de solvente utilizado com diferentes polaridades, a concentração de cada extrato e o número de re-extrações (ROCHA et al., 2013; DAMIANI et al., 2011).

Muitos autores relataram uma correlação positiva entre o elevado valor de atividade antioxidante e o conteúdo de fenólicos totais, quando analisaram frutas (SANTOS et al., 2008; KUSKOSKI et al., 2005).

O extrato da polpa pasteurizada apresentou valor próximo de compostos fenólicos ao do extrato da polpa in natura (Tabela 2), mostrando que a pasteurização não exerceu efeito sobre o teor de compostos fenólicos. O resultado encontrado para o extrato da polpa in natura corrobora com o valor encontrado por Damiani et al. (2011), de 211,50 mg GAE/100 g em extrato hidroetanólico.
O extrato da polpa de araticum apresentou uma elevada quantidade de compostos fenólicos, quando comparado com o extrato de polpas de outras frutas comumente consumidas, como maracujá (20 mg GAE/100 g), cupuaçu (20,5 mg GAE/100 g), graviola (84,3 mg GAE/100 g), uva (117,1 mg GAE/100 g), goiaba (83,0 mg GAE/100 g) (Kuskoski et al., 2005).

Rufino et al. (2010) analisaram o teor de compostos fenólicos de extrato de frutas do Brasil utilizando o mesmo método de extração aplicado neste estudo (extrato combinado metanol-acetona) e obtiveram bons resultados, como o bacuri, a acerola e a carnaúba, que obtiveram valores de 1365; 10280 e 830 mg GAE/100 g, respectivamente.

Para os teores de carotenoides, pode-se observar que o tratamento térmico diminuiu os valores de $\alpha$-caroteno, enquanto que os de $\beta$-caroteno foram maiores na polpa pasteurizada $(8,21 \mu \mathrm{g} / \mathrm{g}$ ) (Tabela 2). Cardoso et al. (2013) investigaram o conteúdo de carotenoides presente na polpa de araticum e encontraram $\alpha$-caroteno (2,98 mg/100 g); $\beta$-caroteno (1,97 mg/100 g) e licopeno (0,02 mg/100 g).

Esse aumento no conteúdo de carotenoides na polpa pasteurizada pode ter sido influenciado por vários fatores decorrentes de processos complexos que afetam a concentração desses compostos, entre os quais o processo de pasteurização. Pode-se citar, como exemplo, o balanço positivo em que a conversão de outros carotenoides a $\alpha$-caroteno e $\beta$-caroteno pode superar as reações de degradação (SILVA et al., 2015).

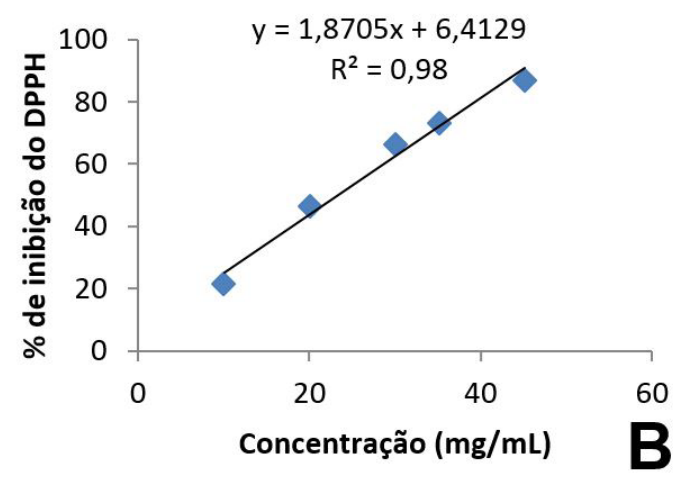

Figura 2. Percentual de atividade antioxidante radicalar em função da concentração do extrato combinado metanol-acetona da polpa de araticum (A) polpa in natura e (B) polpa pasteurizada.

Tabela 2. Teores de compostos fenólicos, atividade antioxidante e carotenoides em polpa de araticum (Annona crassiflora) in natura e pasteurizada, com suas respectivas médias \pm desvio padrão.

\begin{tabular}{|c|c|c|}
\hline Parâmetros & Polpa in natura & Polpa pasteurizada \\
\hline $\mathrm{DPPH}\left(\mathrm{IC}_{50} \mathrm{mg} / \mathrm{mL}\right)$ & $37,59 \pm 0,01$ & $23,30 \pm 0,01$ \\
\hline Compostos fenólicos totais (mg GAE/100 g) & $221,61 \pm 2,03$ & $207,18 \pm 2,05$ \\
\hline$\beta$ caroteno $(\mu \mathrm{g} / \mathrm{g})$ & $4,54 \pm 0,69$ & $8,21 \pm 0,49$ \\
\hline$\alpha$ caroteno $(\mu \mathrm{g} / \mathrm{g})$ & $6,92 \pm 0,17$ & $4,97 \pm 0,11$ \\
\hline
\end{tabular}

GAE: ácido gálico equivalente. 
Tabela 3. Composição centesimal e valor energético de polpa de araticum (Annona crassiflora) pasteurizada, com suas respectivas médias \pm desvio padrão.

\begin{tabular}{lc}
\multicolumn{1}{c}{ Parâmetros $\mathbf{( g / 1 0 0 ~} \mathbf{g})$} & Polpa pasteurizada \\
\hline Umidade & $82,38 \pm 0,04$ \\
Cinzas & $0,42 \pm 0,11$ \\
Proteínas & $1,12 \pm 0,09$ \\
Lipídeos & $1,06 \pm 0,07$ \\
Açúcar redutor em glicose & $13,87 \pm 0,16$ \\
Açúcar não redutor em sacarose & $5,24 \pm 0,91$ \\
Açúcares totais & $19,10 \pm 0,77$ \\
Valor energético $(\mathrm{kcal} / 100 \mathrm{~g})$ & 74,14 \\
\hline
\end{tabular}

Os carotenoides desempenham um papel importante na alimentação humana, pois podem atuar como antioxidantes e apresentar atividade pró-vitamínica $A$, propriedades estas atribuídas especialmente aos $\alpha$-e $\beta$-carotenos e à $\beta$-criptoxantina.

Silva et al. (2015) estudaram o efeito do branqueamento, da pasteurização e do congelamento sobre o teor de carotenoides na polpa de araticum e concluíram que o branqueamento/congelamento a $-5^{\circ} \mathrm{C}$ foi eficaz em até 90 dias de armazenamento, enquanto a pasteurização prolongou o armazenamento em até 180 dias e aumentou o teor de carotenoides em até 6,5\% em relação ao tempo zero (polpa não congelada); dessa forma, a pasteurização torna-se mais eficaz na inibição da velocidade das reações de degradação.

Na literatura, existem poucos relatos de comparação da análise físico-química de polpas pasteurizadas, principalmente sobre frutos do Cerrado, apesar de a pasteurização ser um método muito utilizado em polpas de frutas, melhorando seu estado de conservação (Tabela 3). Comparando-se os valores de açúcares totais do araticum com o PIQ observado para graviola (BRASIL, 2000), a polpa pasteurizada de araticum apresentou valores de açúcares totais mais elevados que os valores máximos permitidos pela legislação e tais valores estão correlacionados com os teores de sólidos solúveis.

Os valores de umidade e proteína encontrados neste experimento apresentaram valores elevados quando comparados com os valores descritos por Silva et al. (2009), de 74,0 g/100 g de umidade e 1,10 g/100 g de proteína, em amostras de araticum que foram submetidas ao processo de branqueamento. Já o conteúdo de lipídeos e cinzas relatados no presente estudo está abaixo dos valores encontrados por Silva et al. (2009), de 1,22 g/100 g e 0,76 g/100 g, respectivamente.

Apesar dessas variações, que dependem de vários fatores, a polpa de araticum pode ser utilizada tanto pela indústria como pelo consumidor, sem perder seu valor nutricional e mantendo sua qualidade nutricional.

\section{Conclusão}

Os resultados mostraram que as polpas apresentam características nutricionais desejáveis, pois se destacaram pelo seu conteúdo de compostos fenólicos, carotenoides e propriedades antioxidantes. O processo de pasteurização mostrou-se um processo de conservação adequado, uma vez que mantém as características físico-químicas e não há perdas acentuadas na composição nutricional, podendo, assim, ser um grande potencial para as indústrias no desenvolvimento de novos produtos.

\section{Agradecimentos}

À Coordenação de Aperfeiçoamento de Pessoal de Nível Superior - CAPES, pela concessão da bolsa de estudos (mestrado e PNPD), ao Conselho Nacional de Desenvolvimento Científico e Tecnológico (CNPq) e ao Instituto de Educação Ciência e Tecnologia de Mato Grosso - IFMT, pelo financiamento do projeto.

\section{Referências}

ALMEIDA, S. P.; COSTA, T. S. A.; SILVA, J. A. Frutas nativas do Cerrado: caracterização físico-química e fonte potencial de nutrientes. In: SANO, S. M.; ALMEIDA, S. P.; RIBEIRO, J. F. (Ed.). Cerrado: ecologia e flora. Brasília: Embrapa Informação Tecnológica, 2008. p. 351-381.

ARÉVALO-PINEDO, A.; CARNEIRO, B. L. A.; ZUNIGA, A. D. G.; ARÉVALO, Z. D. S.; SANTANA, A. A.; PINEDO, R. A. Alterações físico-químicas e colorimétricas de geléias de araticum (Annona Classiflora). Revista Brasileira de Produtos Agroindustriais, v. 15, n. 4, p. 397-403, 2013. http://dx.doi.org/10.15871/15178595/rbpa.v15n4p397-403.

BIANCO, S.; PITELLI, R. A. Fenologia de quatro espécies de frutíferas nativas dos Cerrados de Selvíria, MS. Pesquisa Agropecuária Brasileira, v. 21, n. 11, p. 1229-1232, 1986.

BRAGA FILHO, R. J.; NAVES, R. V.; CHAVES, L. J.; PIRES, L. L.; MAZON, L. T. Caracterização física e físico-química de frutos de araticum (Annona crassiflora Mart.). Bioscience Journal, v. 30, n. 1, p. 16-24, 2014.

BRASIL. Ministério da Agricultura do Abastecimento. Instrução Normativa $n^{\circ} 1$, de 7 de janeiro de 2000. Regulamento técnico geral para fixação dos padrões de identidade e qualidade para polpa de fruta. Diário Oficial [da] República Federativa do Brasil, Brasília, DF, 10 jan. 2000. Seção I, p. 54-58.

CARDOSO, L. M.; OLIVEIRA, D. S.; BEDETTI, S. F.; MARTINO, H. S. D.; PINHEIRO-SANT'ANA, H. M. Araticum (Annona crassiflora Mart.) from the Brazilian Cerrado: chemical composition and bioactive compounds. Food Research International, v. 68, p. 121-134, 2013.

CHITARRA, M. I. F.; CHITARRA, A. B. Pós-colheita de frutas e hortaliças: fisiologia e manuseio. Lavras: UFLA, 2005. 785 p. 
Compostos bioativos e características físico-químicas de polpa de araticum in natura e pasteurizada

Morais, E. C. et al.

DAMIANI, C.; VILAS BOAS, E. V. B.; ASQUIERI, E. R.; LAGE, M. E.; OLIVEIRA, R. A.; SILVA, F. A.; PINTO, D. M.; RODRIGUES, L. J.; SILVA, E. P.; PAULA, N. R. R. Characterization of fruits from the savanna: Araça (Psidium guinnensis Sw.) and Marolo (Annona crassiflora Mart.). Ciência e Tecnologia de Alimentos, v. 31, n. 3, p. 723-729, 2011. http://dx.doi.org/10.1590/S010120612011000300026

ELEZ-MARTínEZ, P.; MARTíN-BELLOSO, O. Effects of high intensity pulsed electric field processing conditions on vitamin $\mathrm{C}$ and antioxidant capacity of orange juice and gazpacho, a cold vegetable soup. Food Chemistry, v. 102, n. 1, p. 201-209, 2007. http://dx.doi.org/10.1016/j.foodchem.2006.04.048.

FRANCO, B. D. G. M.; LANDGRAF, U. Microbiologia dos alimentos. São Paulo: Atheneu, 2008.

GREGORIS, E.; LIMA, G. P. P.; FABRIS, S.; BERTELLE, M.; SICARI, M.; STEVANATO, R. Antioxidant properties of Brazilian tropical fruits by correlation between different assays. BioMed Research International, v. 2013, p. 1-8, 2013. PMid:24106692. http://dx.doi.org/10.1155/2013/132759.

HINNEBURG, I.; DAMIEN, H. J.; RAIMO, H. Antioxidant activities of extracts from selected culinary herbs and spices. Food Chemistry, v. 97, n. 1, p. 122-129, 2006. http://dx.doi. org/10.1016/j.foodchem.2005.03.028.

INSTITUTO ADOLFO LUTZ - IAL. Métodos físico-químicos para análise de alimentos. 4. ed. São Paulo: IAL, 2008. 1020 p.

JAYAPRAKASHA, G. K.; RAO, L. J. Phenolic constituents from lichen Parmotrema stuppeum (Nyl.). Hale and their antioxidant activity. Zeitschrift Naturforsch, v. 55, n. 11-12, p. 1018-1022, 2000. PMid:11204179.

KUSKOSKI, E. M.; ASUERO, G. A.; TRONCOSO, A. M.; MANCINIFILHO, J.; FETT, R. Aplicación de diversos métodos químicos para determinar actividad antioxidante em pulpa de frutos. Ciência e Tecnologia de Alimentos, v. 25, n. 4, p. 726-732, 2005. http://dx.doi.org/10.1590/S0101-20612005000400016.

LATIMER JUNIOR, G. W. (Ed.). Official methods of analysis. 19th ed. Maryland: AOAC International, 2012.

LEE, H. S.; COATES, G. A. Effect of thermal pasteurization on Valencia orange juice color and pigments. Food Science and Technology, v. 36, p. 153-156, 2003.

MERRIL, A. L.; WATT, B. K. Energy value of foods: basis and derivation. Washington: United States Department of Agriculture, 1973. 105 p. (Agriculture Handbook, 74)

MOSCA, J. L.; CAVALCANTE, C. E. B.; DANTAS, T. M. Características botânicas das principais anonáceas e aspectos fisiológicos de maturação. Fortaleza: Embrapa Agroindústria Tropical, 2006. 28 p. (Documentos, 106).

NEVES, L. C. Frutos: o remédio do futuro! Revista Brasileira de Fruticultura, v. 34, n. 4, p. 1, 2012. http://dx.doi.org/10.1590/ S0100-29452012000400042.
OBANDA, M.; OWUOR, P. O.; TAYLOR, S. J. Flavanol composition and caffeine content of green leaf as quality potential indicators of Kenyan Black Teas. Journal of the Science of Food and Agriculture, v. 74, n. 2, p. 209-215, 1997. http://dx.doi.org/10.1002/ (SICI)1097-0010(199706)74:2<209::AID-JSFA789>3.0.CO;2-4.

PACHECO, S.; GODOY, R. L. O.; NASCIMENTO, L. S. M.; CUNHA, C. P.; SANTIAGO, M. C. P. A.; ROSA, J. S. Adaptação do método de extração de carotenóides para escala de micro-extração. In: REUNIÃO DE BIOFORTIFICAÇÃO, 4., 2011, Teresina, Piauí. Palestras e Resumos... Rio de Janeiro: Embrapa Agroindústria de Alimentos; Teresina: Embrapa Meio-Norte, 2011. 1 CD-ROM.

PIMENTA, A. C.; SILVA, P. S. R.; ZUFFELLATO-RIBAS, K. C.; KOEHLER, H. S. Caracterização de plantas e de frutos de araticunzeiro (Annona crassiflora Mart.) nativos no Cerrado Mato-grossense. Revista Brasileira de Fruticultura, v. 36, n. 4, p. 892-899, 2014. http://dx.doi.org/10.1590/0100-2945-416/13.

PLAZA, L.; SÁNCHEZ-MORENO, C.; ELEZ-MARTÍNEZ, P.; ANCOS, B.; MARTíN-BELLOSO, O.; CANO, M. P. Effect of refrigerated storage on vitamin $\mathrm{C}$ and antioxidant activity of orange juice processed by high-pressure or pulsed electric fields with regard to low pasteurization. European Food Research and Technology, v. 223, n. 4, p. 487-493, 2006. http://dx.doi.org/10.1007/s00217005-0228-2.

RAMOS, E. M.; GOMIDE, L. A. M. Avaliação da qualidade de carnes: fundamentos e metodologias. Viçosa: Ed. UFV, 2007. $599 \mathrm{p}$.

ROCHA, M. S.; FIGUEIREDO, R. W.; ARAÚJO, M. A. M.; MOREIRAARAÚJO, R. S. R. Caracterização físico-química e atividade antioxidante (in vitro) de frutos do cerrado Piauiense. Revista Brasileira de Fruticultura, v. 35, n. 4, p. 933-941, 2013. http:// dx.doi.org/10.1590/S0100-29452013000400003.

ROESLER, R.; MALTA, L. G.; CARRASCO, L. C.; HOLANDA, R. B.; SOUSA, C. A. S.; PASTORE, G. M. Atividade antioxidante de frutas do cerrado. Ciência e Tecnologia de Alimentos, v. 27 , n. 1, p. 53-60, 2007. http://dx.doi.org/10.1590/S010120612007000100010.

RUFINO, M. S. M.; ALVES, R. E.; DE BRITO, E. S.; PÉREZ-JIMÉNEZ, J.; SAURA-CALIXTO, F.; MANCINI-FILHO, J. Bioactive compounds and antioxidant capacities of 18 non-traditional tropical fruits from Brazil. Food Chemistry, v. 121, n. 4, p. 996-1002, 2010. http://dx.doi.org/10.1016/j.foodchem.2010.01.037.

SALES, A.; WAUGHON, T. G. M. Influência do processamento no teor de compostos bioativos em frutos de murici e cajá. Revista Agrarian, v. 6, n. 19, p. 7-15, 2013.

SANTOS, G. M.; MAIA, G. A.; SOUSA, P. H. M.; COSTA, J. M. C. C.; FIGUEIREDO, R. W.; PRADO, G. M. Correlação entre atividade antioxidante e compostos bioativos de polpas comerciais de açaí (Euterpe oleracea Mart). Archivos Latinoamericanos de Nutricion, v. 58, n. 2, p. 187-192, 2008. PMid:18833997. 
Compostos bioativos e características físico-químicas de polpa de araticum in natura e pasteurizada Morais, E. C. et al.

SILVA, A. M. L.; GOMES, A. C. G.; MARTINS, B. A. Alterações físico-químicas e estudo enzimático da polpa de araticum (Annona crassiflora Mart). Estudos, v. 36, n. 5-6, p. 775-783, 2009.

SILVA, L. L.; CARDOSO, L. M.; PINHEIRO-SAN'TANA, H. M. Influência do branqueamento, pasteurização e congelamento nas características físico-químicas e carotenoides de polpa de araticum. Boletim Centro de Pesquisa de Processamento de Alimentos, v. 33, n. 1, p. 49-59, 2015.

SOUZA-SARTORI, J. A.; SCALISE, C.; BAPTISTA, A. S.; LIMA, R. B.; AGUIAR, C. L. Parâmetros de influência na extração de compostos fenólicos de partes aéreas da cana de-açúcar com atividade antioxidante total. Bioscience Journal, v. 29, n. 2, p. 297-307, 2013. 